\title{
Role of tensor terms of the Skyrme energy-density functional on neutron deformed magic numbers in the rare-earth region
}

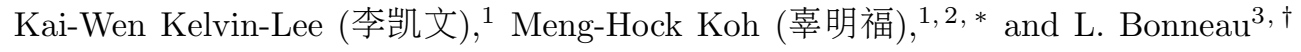 \\ ${ }^{1}$ Department of Physics, Faculty of Science, Universiti Teknologi Malaysia, 81310 Johor Bahru, Johor, Malaysia \\ ${ }^{2}$ UTM Centre for Industrial and Applied Mathematics, 81310 Johor Bahru, Johor, Malaysia \\ ${ }^{3}$ CENBG, UMR 5797, Université de Bordeaux, CNRS, F-33170, Gradignan, France
}

(Dated: February 26, 2021)

\begin{abstract}
The role of the tensor part of the nuclear interaction is actively investigated in recent years due to experimental advancement yielding new data in nuclei far from the $\beta$-stability line. In this article we study the effect of this part of the nuclear interaction on deformed neutron magic numbers in the rare-earth region within the Skyrme energy-density functional for various TIJ [1] parametrizations. Two quantities signaling magic numbers are considered: two-neutron separation energies and singleparticle energies. They are calculated in isotopic series involving well-deformed rare-earth nuclei ranging from $Z=64$ to $Z=72$ in the $N=100$ region. Obtained results show that, whereas the neutron-proton tensor contribution to binding energies is important to reproduce neutron sub-shell closure at $N=104$ in heavier rare earths $\mathrm{Yb}(Z=70)$ and $\mathrm{Hf}(Z=72)$ isotopes, like-particle tensor also plays a role in the single-particle spectrum around Fermi level and is even favored in lighter Gd $(Z=64)$ and Dy $(Z=66)$ rare-earth isotopes.
\end{abstract}

\section{INTRODUCTION}

The discovery of gravitational-wave signal GW170817 coming from binary neutron stars (BNS) merger shows that BNS merger is more likely the candidate site for rapid neutron capture process (known as the $r$ process) [2]. The nucleosynthesis through $r$ process occurs through rapid capture of free neutrons forming neutronrich elements away from the beta-stability line. In the solar abundances, the $r$ process is responsible for the second and third peaks around $A=130 \sim 138$ and $A=195 \sim 208$, respectively 3 . These peaks are attributed to neutron shell closures giving rise to relatively high stability. A small peak is also observed around $A=165$ in the rare-earth region. This peak was explained in terms of increasing nuclear deformation which stabilizes the nucleus similarly to the role of neutron closed shell [4. Substantial effort has been made by the experimental nuclear physics community to uncover this so-called deformed magic numbers. However, as we will see shortly, these magic numbers remain somewhat elusive.

On the experimental side, considerable effort has been made resulting in many proposals for new deformed magic numbers. In 1999, Asai and collaborators showed two minimum in the first $2^{+}$energies in Dy isotopes [5]. They proposed that the second minima at $N=104 \mathrm{co}-$ incides with the location of maximum deformation. The first minimum at $N=98$ was however dismissed as arising due to some local effect which enhanced the deformation around this isotope. This conclusion on $N=98$ was also agreed upon in the work of [6] about a decade later based on systematic studies on yrast levels of Dy

\footnotetext{
* kmhock@utm.my

$\dagger$ bonneau@cenbg.in2p3.fr
}

isotopes and the $4^{+} \rightarrow 2^{+}$transition in ${ }^{170}$ Dy. Subsequent work by Patel et al. [7] in 2014 showed that a deformed magic number exists for neutrons at $N=100$ in elements with proton number $Z \leq 66$, namely in $\mathrm{Nd}$ $(Z=60), \operatorname{Sm}(Z=62), \operatorname{Gd}(Z=64)$ and Dy $(Z=66)$ isotopes. This magic-number character of $N=100$ in this region was, however, challenged three years later by $\mathrm{Wu}$ and collaborators 8 . They reported to find no evidence of deformed subshell gap at $N=100$ from the analyses of $\beta$ - decay half-lives of $\operatorname{Pm}(Z=61)$ isotopes. Instead, they proposed two different magic numbers at $N=96$ for $Z=58$ to $Z=62$ and $N=104$ for $Z=63$ to $Z=66$. The more recent works of Hartley and collaborators 9,10 on the other hand showed that $N=98$ could instead be a candidate neutron subshell closure around $Z=64$, contradicting the findings of Wu et al. 8 that $N=104$ should be the deformed magic number in this isotope. Going to heavier rare-earth nuclei, 11 reported emergence of new sub-shell closure at $N=108$ in ${ }_{72} \mathrm{Hf}$ $(Z=72), \mathrm{W}(Z=74)$ and $\mathrm{Os}(Z=76)$ isotopic series.

On the theoretical side, there are rather limited studies to uncover the possible deformed magic numbers in the rare-earth region. To the best of our knowledge, all of the studies supported the magicity of $N=100$ in the light rare-earth elements Sm and/or Dy [12 14]. The possibility of different subshell closures in heavier rare-earth isotopes $(Z>66)$ as indicated by some experimental data was not explored. There were however calculations on $K$ isomers for example the $6^{+}$in ${ }^{170}$ Dy [15, 16] and yrast levels in Dy isotopes [17. Interestingly, Yadav et al. 17] reported that $N=102$ is more likely the magic number in Dy isotopes instead of $N=104$ based on energies of the ground-state and first $2^{+}$states obtained within cranked Hartree-Fock-Bogoliubov calculations.

One of the current major theme in nuclear theory is related to the impact of tensor two-nucleon interaction. While pioneering work on tensor effective potential was performed in 1977 by Stancu et al. [18, there was not 
much follow up of this work until mid 2000s when access to exotic nuclei was made possible through technological and experimental breakthroughs. Within the mean-field approach based on Skyrme energy-density functional, efforts have been made to design new parametrizations through either a perturbative or a full fitting procedure. In the perturbative approach, only the two zero-range tensor terms are adjusted while all other Skyrme parameters are kept constant. This is the case for the SIII+tensor (SIII+T) parameter sets of Refs. [18, 19] and the SLy5+tensor (SLy5+T) parametrization of Ref. [20]. On the other hand, a fit of all parameters has been performed by Lesinski et al. [1] yielding a set of TIJ parametrizations which were applied to the investigations of spherical nuclei 1, nuclear deformation 21] and time-odd systems [22]. Investigations on fit protocols of tensor effective potential components was also studied by Zalewski et al. 23] who proposed that the singleparticle levels should be considered instead of the usual bulk properties like the binding energies.

Within the Gogny mean-field approach, similar effort has been made by Anguiano et al. 24] highlighting the need for inclusion of tensor effective potential. In this work a density-independant, finite-range tensor interaction term is added to the D1S parametrization, yielding the parametrization called D1ST2a. In this perturbative approach, independant like-nucleon and neutronproton contributions are present in the effective twonucleon potential, as in the zero-range Skyrme tensor potential, and the fitting protocol-involving neutron singleparticle energies of $1 f_{5 / 2}$ and $1 f_{7 / 2}$-yielded a strength of $-20 \mathrm{MeV}$ for the like-nucleon term and a much larger strength of $115 \mathrm{MeV}$ for the neutron-proton term. Subsequently Grasso and Anguiano [25] studied the appropriate range for the strength of the tensor terms within the Skyrme and Gogny energy density functionals (EDF) while Ref. 26] showed that tensor effective potential is important to explain magicity at $N=32$ and $N=34$ in the ${ }^{52} \mathrm{Ca}$ and ${ }^{54} \mathrm{Ca}$ nuclei. More recently, Bernard and collaborators [27] investigated the role in fission of the tensor terms of the Gogny EDF through a thorough comparison of several fission-related quantities-ranging from fission-barrier heights and paths to fission-fragment neutron emission-obtained with the D1S and D1ST2a parametrizations. One of the most important conclusion is that the added tensor terms are able to account for the new compact-symmetric fission configuration experimentally observed during the 2012 SOFIA campaign at GSI Darmstadt [28].

From the rich literature showing that a tensor effective potential affects the single-particle levels ordering, we are interested to investigate if inclusion of such a potential within our Skyrme EDF would allow us to explain deformed magic numbers suggested by experiment in the rare-earth region. We shift our attention to the heavier rare-earth nuclei which have not gained much attention from theorists as compared to their lighter counterparts with particular interest in the $N=104$ sub-shell closures.
After a brief presentation of the relevant theoretical ingredients, we address successively in sections III to $\mathrm{V}$ the effect of the Skyrme tensor effective potential on charge quadrupole moments, two-neutron separation energies, and single-particle spectra. We give concluding remarks in section VI.

\section{THEORETICAL APPROACH}

We considered several Skyrme fully refitted TIJ parametrizations namely

- T22, T24 and T26 (pure like-particle coupling)

- T22, T42 and T62 (pure neutron-proton coupling)

- T41 and T44 (mixed coupling).

The TIJ forces are labelled in such a way that $I$ and $J$ values are related to the proton-neutron $\beta$, and likeparticle $\alpha$ tensor coupling, respectively [1]

$$
\begin{aligned}
& \alpha=60(J-2) \mathrm{MeV} \mathrm{fm}^{3} \\
& \beta=60(I-2) \mathrm{MeV} \mathrm{fm}^{3}
\end{aligned}
$$

with $\alpha=\alpha_{C}+\alpha_{T}$ and $\beta=\beta_{C}+\beta_{T}$. The subscript $\mathrm{C}$ and $\mathrm{T}$ refer to the central and tensor contributions, respectively.

We have also included the original SIII 29] and SLy5 30. parametrizations and their counterparts in which tensor effective potential components are added perturbatively for comparison. The seniority force is used to approximate the residual pairing interaction whereby the neutron and proton pairing strengths were adjusted such that the BCS pairing gap yields the empirical Jensen formula 31. The single-particle wave function is expanded on a deformed harmonic oscillator basis with a basis size of 16. The oscillator parameters $b$ and $q$ have been optimized to yield the lowest ground-state energy for each nucleus 32]. We have limited ourselves to axial and parity symmetric nuclear shapes.

Within the Skyrme EDF, in addition to the strength parameters of the central and spin parts of the effective potential, the tensor parameters $t_{e}$ and $t_{o}$ enter the total binding energy in the $B_{14}, B_{15}, B_{16}$ and $B_{17}$ coupling constants given by

$$
\begin{aligned}
& B_{14}=-\frac{t_{1} x_{1}+t_{2} x_{2}}{8}+\frac{1}{4}\left(t_{e}+t_{o}\right) \\
& B_{15}=\frac{t_{1}-t_{2}}{8}-\frac{1}{4}\left(t_{e}-t_{o}\right) \\
& B_{16}=-\frac{3}{8}\left(t_{e}+t_{o}\right) \\
& B_{17}=\frac{3}{8}\left(t_{e}-t_{o}\right) .
\end{aligned}
$$

In order to isolate the contribution from the tensor parameters to the binding energy, we separate the contributions coming from $B_{14}$ and $B_{15}$ into two parts, such 
that:

$$
\begin{aligned}
& E_{B_{14}}^{C}=-\left(\frac{t_{1} x_{1}+t_{2} x_{2}}{8}\right) \mathbf{J}_{\mu \nu} \mathbf{J}_{\mu \nu} \\
& E_{B_{14}}^{T}=\frac{1}{4}\left(t_{e}+t_{o}\right) \sum_{\mu, \nu=x}^{z} \mathbf{J}_{\mu \nu} \mathbf{J}_{\mu \nu} \\
& E_{B_{15}}^{C}=\left(\frac{t_{1}-t_{2}}{8}\right) \sum_{\mu, \nu=x}^{z} \mathbf{J}_{q, \mu \nu} \mathbf{J}_{q, \mu \nu} \\
& E_{B_{15}}^{T}=-\frac{1}{4}\left(t_{e}-t_{o}\right) \sum_{\mu, \nu=x}^{z} \mathbf{J}_{q, \mu \nu} \mathbf{J}_{q, \mu \nu}
\end{aligned}
$$

where $\mathbf{J}_{\mu \nu}$ is the spin-current density with $\mu, \nu=\{r, z, \phi\}$ and $\mathbf{J}_{q, \mu \nu}$ is the spin-current density for the charge state $q$ (see Ref. 33 for their definition). The contributions from the $B_{16}$ and $B_{17}$ terms to the binding energy are

$$
\begin{aligned}
& E_{B_{16}}=B_{16} \sum_{\mu=x}^{z}\left(\mathbf{J}_{\mu \mu}\right)^{2} \\
& E_{B_{17}}=B_{17} \sum_{\mu=x}^{z}\left(\mathbf{J}_{q, \mu \mu}\right)^{2} .
\end{aligned}
$$

Separating these terms in such a way allows us to draw out the contribution of the tensor part alone from all other non-tensor related terms to the binding energy. This means that the binding energy can be partitioned into

$$
E=E_{C}+E_{T}
$$

where

$$
E_{C}=E_{k i n}+E_{\text {Coul }}+E_{\text {pair }}+E_{B_{x}}+E_{B_{14}}^{C}+E_{B_{15}}^{C}
$$

with the contribution to the $E_{B_{x}}$ term comes from all the Skyrme coupling constants except for $B_{14}, B_{15}, B_{16}$ and $B_{17}$. The contribution to the $E_{T}$ comes from terms related to the tensor effective potential parameters $t_{e}$ and $t_{o}$ such that

$$
E_{T}=E_{B_{14}}^{T}+E_{B_{15}}^{T}+E_{B_{16}}^{C}+E_{B_{17}}^{C} .
$$

\section{CHARGE INTRINSIC QUADRUPOLE MOMENT}

We first present our ground-state intrinsic charge quadrupole moment for isotopic series of ${ }_{64} \mathrm{Gd},{ }_{66} \mathrm{Dy}$, ${ }_{68} \mathrm{Er},{ }_{70} \mathrm{Yb}$ and ${ }_{72} \mathrm{Hf}$ and compared to experiment [34] in Figure 1. Calculations with the various TIJ forces give good agreement with available experimental data. More importantly, we find a peak around $N \sim 100$, which corresponds to the maximum deformation in the region. The enhanced stability around this neutron number suggests the existence of deformed magic numbers, giving us confidence that our investigation should be centered around $N \sim 100$. Yet, the charge quadrupole moment being a bulk property of the nucleus, it does not allow us to comment more on what are the possible predicted deformed magic numbers nor on the actual role of tensor effective potential. As such, we shift our attention to another observable expected to be more sensitive to a shell gap in the neutron single-particle spectrum, namely the two-neutron seperation energy.

\section{TWO-NEUTRON SEPARATION ENERGIES}

We compute the two-neutron separation energy $S_{2 n}$ and two-neutron separation energy differential $\Delta S_{2 n}$ using the expression

$$
\begin{gathered}
S_{2 n}=E(N-2, Z)-E(N, Z) \\
\Delta S_{2 n}=S_{2 n}(N, Z)-S_{2 n}(N+2, Z) .
\end{gathered}
$$

The calculated $\Delta S_{2 n}$ are plotted in Figure 2 together with experimental data taken from AME2016 [35].

Let us first discuss the results for the three heavier elements considered in our study namely ${ }_{68} \mathrm{Er},{ }_{70} \mathrm{Yb}$ and ${ }_{72}$ Hf. The experimental data show a peak at $N=104$ [35] in these elements. To compare the theoretical results with data, we take the T22 as the reference parametrization because it is such that $\alpha=\beta=0$, although the Skyrme parameters $t_{e}, t_{o}$ are not zero. The T22 parameter set manages to produce a pronounced peak at $N=104$ especially in ${ }_{68} \mathrm{Er}$ and ${ }_{70} \mathrm{Yb}$. The peak at this neutron number is even more enhanced when increasing $\beta$ by considering the T42 and T62 parametrizations. This shows that neutron-proton tensor coupling constant $\beta$ is essential to reproduce the neutron $N=104$ sub-shell closure in these rare-earth nuclei. This behavior of the $N=104$ peak with $\beta$ is even more marked in ${ }_{72} \mathrm{Hf}$ isotopes.

On the contrary, increasing like-particle tensor coupling constant $\alpha$ with a vanishing $\beta$ contribution, i.e. in the sequence $\mathrm{T} 22 \rightarrow \mathrm{T} 24 \rightarrow \mathrm{T} 26$, results in larger dips, instead of peaks, at $N=104$. With non-vanishing $\beta$ and still increasing $\alpha$ in the sequence T41 $\rightarrow$ T42 $\rightarrow$ T44 parametrizations, we see that the pronounced peak at $N=104$ obtained with T41 decreases when using the T42 parametrization, and then vanished totally with T44. This clearly shows that like-particle tensor coupling tends to remove the $N=104$ peak in heavy rare-earth nuclei. Therefore the reproduction of this peak requires small $\alpha$ values and positive, sizeable $\beta$ values.

Concerning the two parametrizations obtained from perturbative fits of the tensor effective potential, we find that the SLy5+T improves the results as compared to the original SLy5 parametrization. Indeed a significant peak is found with SLy5+T in the $\Delta S_{2 n}$ plot at $N=104$ for ${ }_{70} \mathrm{Yb}$ isotopes instead of a minute peak at $N=102$ with SLy5. However, neither SLy5 nor SLy5+T are able to reproduce the magicity of $N=104$ in ${ }_{72} \mathrm{Hf}$ isotopes. 
Before moving on to lighter elements of the rare-earth region, we make a remark on the $\Delta S_{2 n}$ at $N=108$ in ${ }_{72} \mathrm{Hf}$ isotopes. In this element, the experimental point at $N=108$ is higher than the one at $N=104$. We did not manage to reproduce this pattern in our calculations. In spite of this, we see that the $\Delta S_{2 n}$ between $N=106$ and $N=108$ exhibits a positive slope when using T42 and T62 forces, while all other TIJ forces give a negative slope. This reinforces the conjecture that neutron-proton tensor coupling is more favored in heavy rare-earth nuclei and can, at the very least, reproduce the experimental trend qualitatively.

In ${ }_{66}$ Dy isotopes, peaks are seen at $N=98,102$ and possibly $N=106$ in experimental $\Delta S_{2 n}$ [35]. Calculations with TIJ parametrizations are not able to reproduce this experimental trend. Instead, the SIII and SIII+T parametrizations performed better there. The SIII parametrization generates peaks at $N=98$ and 102 while the SIII+T calculations yield a peak at $N=102$ and follow the experimental trend at $N=106$. Results with the TIJ parameter sets, however, yield two peaks at $N=100$ and $N=104$. The former peak is enhanced when increasing the like-particle coupling constant $\alpha$, while the latter is more pronounced when the neutronproton coupling constant $\beta$ is larger. Indeed, T22 calculations serving as a reference, we see that the peak at $N=100$ is more pronounced when going to T24 and T26 forces. Conversely, the peak at the same neutron number is reduced when going from T22 to T42 and then to T62 forces. The reverse is seen at $N=104$ where going from T22 to T24 induced a sharp drop in the $\Delta S_{2 n}$. Comparing the results obtained with T41, T42 and T44 also indicates that strong like-particle tensor coupling is undesirable to produce a peak at $N=104$, similar to what is found in heavier rare-earth elements. Before closing this discussion of ${ }_{66}$ Dy results, we would like to draw the reader's attention to the fact that while our TIJ calculations do not reproduce experimental data of Wang et al. 35], the TI2 results are however in agreement with $\mathrm{Wu}$ et al. 8] who showed that $N=104$ forms a subshell closure. Clearly more experimental data in light rare-earth nuclei are needed to resolve this discrepancy.

Finally, we comment on the results for the lightest rareearth element considered in our study. In the ${ }_{64} \mathrm{Gd}$ isotopes, the experimental $\Delta S_{2 n}$ is almost constant over the range $94-98$ of $N$. It makes a dip at $N=100$ before forming a peak at $N=102$. Our TIJ calculations fails to reproduce this trend, and the same pattern seen in the variation of the above $N=100$ and $N=104$ peaks with $\alpha$ and $\beta$ is obtained here.

To conclude this section, we can say that the TIJ parametrizations produce two persistent peaks at $N=$ 100 and $N=104$. The peaks can be obtained in particular with the T22 parameter set for which the central and tensor contributions cancel, yielding $\alpha=\beta=0$. When switching to TIJ forces with $\alpha>0$, we find an enhancement for the $N=100$ peak while TIJ forces with $\beta>0$ accentuates the peak at $N=104$.
In order to understand the role of the tensor effective potential, we plot in Figure 3 the contributions of $E_{C}$ and $E_{T}$ terms of the Skyrme energy-density to $\Delta S_{2 n}$ as a function of $N$ for T26, T22 and T62 parametrizations. In all considered nuclei, the tensor contribution is small as compared to the sum of all other terms, but it plays an important role in shaping the fine structure of the patterns seen in Figure 2 .

In ${ }_{72} \mathrm{Hf}$, the tensor contribution is particularly crucial when using T22 and T62 parameter sets. Indeed the contribution from all other terms to $\Delta S_{2 n}$ does not yield a peak at $N=104$, which can only be obtained thanks to the tensor component.

In ${ }_{70} \mathrm{Yb}$, non-tensor terms alone do produce two peaks at $N=100$ and $N=104$ with the T22 and T26 forces. When including tensor, the calculated $\Delta S_{2 n}$ at $N=100$ is decreased while the point at $N=104$ is push upwards yielding only one peak at $N=104$. A similar effect is observed in in ${ }_{64} \mathrm{Gd}$ and ${ }_{66}$ Dy elements.

\section{NEUTRON SINGLE-PARTICLE ENERGY SPECTRA}

We now turn our attention to the neutron singleparticle levels for some nuclei in Figure 4. The variation in the calculated $\Delta S_{2 n}$ with different Skyrme parametrizations in Figure 2 coincide with the variation in the single-particle energy gap.

In ${ }_{66} \mathrm{Dy}$ isotopes, two pieces of information can be learnt. On the one hand pure like-particle $(\alpha)$ tensor coupling (as in T24 and T26) favors sub-shell closure at $N=100$. In fact, a substantial single-particle energy gap appears at $N=96$ only with T26 force. Pure neutronproton $(\beta)$ tensor coupling, on the other hand, favors sub-shell closure at $N=104$. The $N=98$ sub-shell closure, while not reproduced by any TIJ forces, seems to be accounted for by a strong neutron-proton rather than like-particle tensor component. This is correlated with the decreasing trend in the single-particle energy gap at $N=98$ when going from T24 to T26. However, according to Hartley et al in Ref. [9], "1/2 neutron orbital above the $7 / 2$ one is required to explain the decay properties of ${ }_{63}^{162} \mathrm{Eu}$ ". This suggests to explore refinements to existing parametrizations.

Let us move to the single-particle states of ${ }_{70} \mathrm{Yb}$ and ${ }_{72}$ Hf isotopes. The peak in $\Delta S_{2 n}$ at $N=104$ is related to the widening of the single-particle energy gap between the $7 / 2^{-}$and $7 / 2^{+}$states seen in Figure 4 . The energy gap between the two states increases with $\alpha$ (along the sequence of calculations $\mathrm{T} 22 \rightarrow \mathrm{T} 42 \rightarrow \mathrm{T} 62)$, while it decreasing from T24 to T26, that is to say when $\alpha=0$ and $\beta$ increases. With T22, we see that the $7 / 2^{+}$state is located below a $5 / 2^{-}$state and remains almost at the same energy when switching on neutron-proton tensor coupling and keeping $\alpha=0$. In contrast the $7 / 2^{-}$state keeps beeing shifted higher in energy, giving rise to a very large energy gap when increasing neutron-proton tensor 
strength.

Moreover an important observation is made regarding the like-particle tensor coupling by comparing the results obtained with T42 and T44 forces. A slight increase of like-particle tensor coupling in T44 causes tremendous lowering of the $7 / 2^{-}$state, while elevating the $7 / 2^{+}$state above $5 / 2^{-}$. Consequently, the $N=104$ is not a subshell closure for T44 while it is so for T42. This suggests that strong neutron-proton coupling is important to reproduce this deformed magic number in the heavy rare-earth region. However, the increasing energy gap at $N=106$ sub-shell in ${ }_{70} \mathrm{Yb}$ and ${ }_{72} \mathrm{Hf}$ isotopes with T24 and T26 could indicate the importance of like-particle tensor coupling.

\section{CONCLUSION}

In conclusion, we have performed Skyrme HartreeFock-BCS calculations for even-even rare-earth nuclei with $Z=64$ up to $Z=72$. We have found a maximum deformation around $N \sim 100$ which confirms that the neutron deformed magic numbers could be found in this neutron-number region.

Then we have calculated two-neutron separation energies and studied their difference $\Delta S_{2 n}$ between two consecutive even- $N$ values. with several TIJ parametrizations of the Skyrme energy-density functional. Two persistent peaks have been found at $N=100$ and $N=104$. These peaks have been obtained in all considered nuclei with the T22 parameter set (for which $\alpha=\beta=0$ ) except for ${ }_{72} \mathrm{Hf}$. While the $N=100$ peak is even more pronounced when switching on like-particle tensor terms (driven by the $\alpha$ coupling constant) the $N=104$ is enhanced by neutron-proton tensor terms (driven by the $\beta$ coupling constant). Comparison with experimental data of Ref. 35] suggests that neutron-proton tensor terms are favored in heavy rare-earth nuclei to reproduce the $N=104$ peak. In contrast, increasing like-particle tensor strength with a fixed, positive $\beta$ coupling constant (in T41, T42, T44 parametrizations) has the detrimental effect of decreasing the $N=104$ peak. In the lighter rareearth elements ${ }_{64} \mathrm{Gd}$ and ${ }_{66} \mathrm{Dy}$ however, the situation is not so clear. In these nuclei the like-particle tensor terms can produce a peak in $\Delta S_{2 n}$ at some neutron numbers depending on the parametrization.

To better understand the role of the tensor terms on this observable, we have studied the contribution to $\Delta S_{2 n}$ arising solely from the $t_{e}$ and $t_{o}$ parameters of the tensor effective potential and shown that, while being small, this contribution is important to produce the $\Delta S_{2 n}$ peaks. We have also studied the neutron single-particle spectra for various parametrizations of the Skyrme EDF. A neat correlation between the peak structure of $\Delta S_{2 n}$ and large single-particle energy gaps around Femi level could thus be evidenced.

Overall the present work indicates that $N=104 \mathrm{can}$ be considered as a "deformed" magic neutron number thanks to neutron-proton tensor coupling in heavy rareearth elements and that like-particle tensor coupling is not desirable in this region. However, in lighter rareearth elements the situation is less clear and further work is called for to better understand the intricate role of tensor terms of the effective nucleon-nucleon potential.

\section{ACKNOWLEDGMENTS}

K.W K.L and M.H.K would like to acknowledge Malaysian Ministry of Education for the financial support through the Fundamental Research Grant Scheme (FRGS/1/2018/ST/G02/UTM/02/6) and UTM (R.J130000.7854.5F028).
[1] T. Lesinski, M. Bender, K. Bennaceur, T. Duguet, and J. Meyer, Phys. Rev. C 76, 014312 (2007).

[2] B. P. Abbott and et al., Astrophys. J. Lett. 850, L39 (2017).

[3] M. Arnould, S. Goriely, and K. Takahashi, Phys. Rep. 450, 97 (2007).

[4] R. Surman, J. Engel, J. R. Bennett, and B. S. Meyer, Phys. Rev. Lett 79, 1809 (1997).

[5] M. Asai and et al., Phys. Rev. C 59, 3060 (1999).

[6] P.-A. Söderström and et al., Phys. Rev. C 81, 034310 (2010).

[7] Z. Patel and et al., Phys. Rev. Lett 113, 262502 (2014).

[8] J. Wu and et al., Phys. Rev. Lett 118, 072701 (2017).

[9] D. Hartley and et al., Phys. Rev. Lett 120, 182502 (2018).

[10] D. Hartley and et al., Phys. Rev. C 101, 044301 (2020).

[11] H. Watanabe, Eur. Phys. J. A 55 (20189).

[12] L. Satpathy and S. K. Patra, J. of Phys. G 30, 771-781 (2004).
[13] S. K. Ghorui, B. B. Sahu, C. R. Praharaj, and S. K. Patra, Phys. Rev. C 85, 064327 (2012).

[14] M. Kaur, A. Quddus, A. Kumar, M. Bhuyan, and S. K. Patra, J. of Phys. G 47, 105102 (2020).

[15] P. Regan, F. Xu, P. Walker, M. Oi, A. Rath, and P. Stevenson, Phys. Rev. C 65, 037302 (2002).

[16] A. Rath, P. Stevenson, P. Regan, F. Xu, and P. Walker, Phys. Rev. C 68, 044315 (2003).

[17] H. Yadav, M. Kaushik, I. Jakhar, and A. Ansari, Part. Nucl. Lett. 112, 66 (2002).

[18] F. Stancu, D. M. Brink, and H. Flocard, Phys. Lett. B 68, 108 (1977).

[19] D. M. Brink and F. Stancu, Phys. Rev. C 97, 064304 (2018).

[20] G. Colo, H. Sagawa, S. Fracasso, and P. F. Bortignon, Phys. Lett. B 646, 227 (2007).

[21] M. Bender, K. Bennaceur, T. Duguet, P. H. Heenen, T. Lesinski, and J. Meyer, Phys. Rev. C 80, 064302 (2009). 
[22] V. Hellemans, P.-H. Heenen, and M. Bender, Phys. Rev. C 85, 014326 (2012).

[23] M. Zalewski, J. Dobaczewski, W. Satuła, and T. R. Werner, Phys. Rev. C 77, 024316 (2008).

[24] M. Anguiano, M. Grasso, G. Co', V. D. Donno, and A. M. Lallena, Phys. Rev. C 86, 054302 (2012).

[25] M. Grasso and M. Anguiano, Phys. Rev. C 88, 054328 (2013).

[26] M. Grasso, Phys. Rev. C 89, 034316 (2014).

[27] R. N. Bernard, N. Pillet, L. M. Robledo, and M. Anguiano, Phys. Rev. C 101, 044615 (2020).

[28] A. C. et al, Phys. Rev. C 99, 054628 (2019).

[29] M. Beiner, H. Flocard, N. V. Giai, and P. Quentin, Nucl. Phys. A 238, 29 (1975).
[30] E. Chabanat, P. Bonche, P. Haensel, J. Meyer, and R. Schaeffer, Nucl. Phys. A 635, 231 (1998).

[31] A. S. Jensen, P. G. Hansen, and B. Jonson, Nucl. Phys. A 431, 393 (1984).

[32] N. M. Nor, N.-A. Rezle, K.-W. Kelvin-Lee, M.-H. Koh, L. Bonneau, and P. Quentin, Phys. Rev. C 99, 064306 (2019).

[33] Y. M. Engel, D. M. Brink, K. Goeke, S. J. Krieger, and D. Vautherin, Nucl. Phys. A 249, 215 (1975).

[34] S. Raman, C. W. N. Jr, and P. Tikkanen, Atomic Data and Nuclear Data Tables 78, 1 (2001).

[35] M. Wang, G. Audi, F. G. Kondev, W. J. Huang, S. Naimi, and X. Xu, Chin. Phys. C 41, 030003 (2017). 

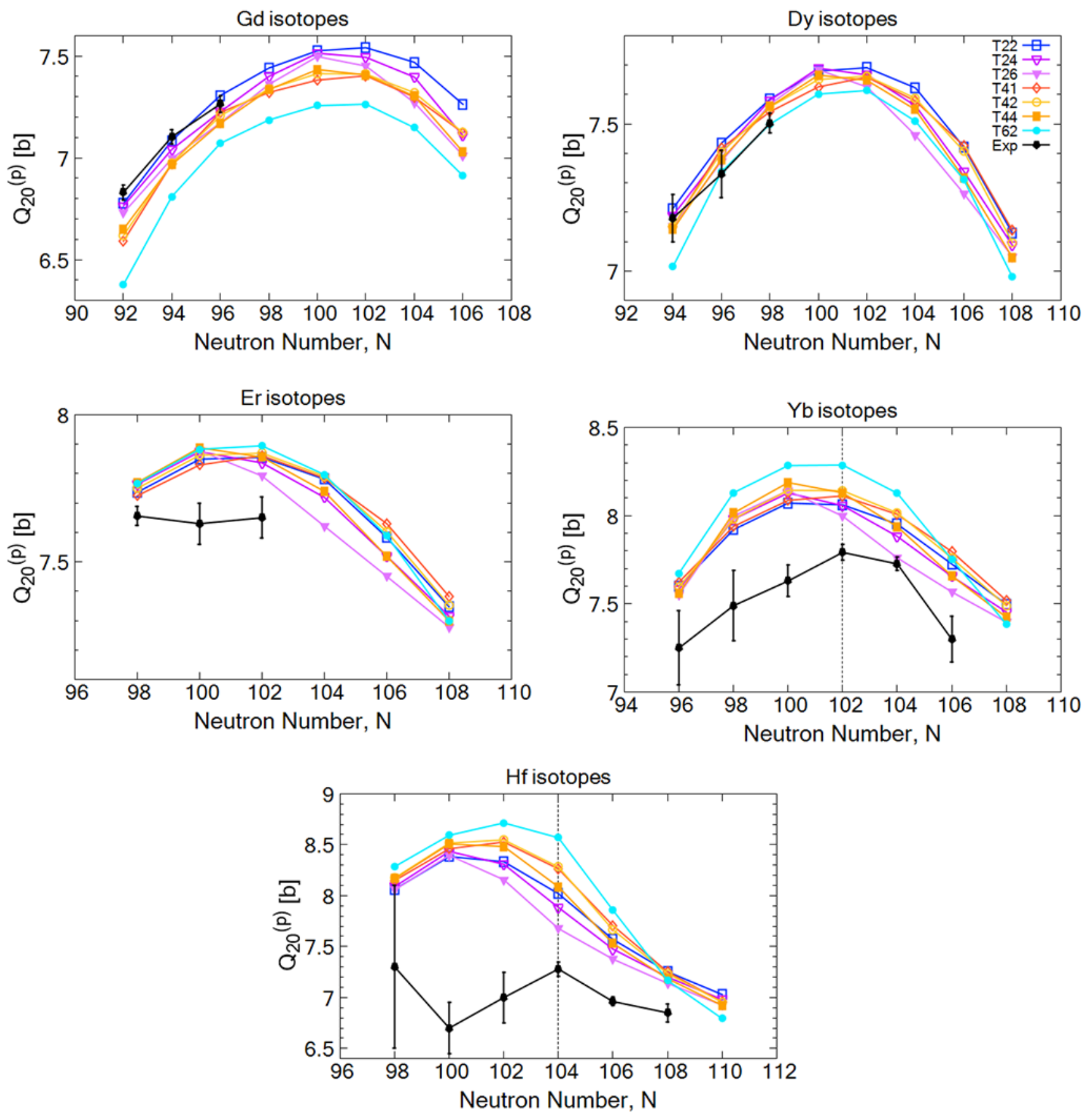

FIG. 1: Intrinsic charge (proton) quadrupole moment obtained with seven TIJ parametrizations. Experiment data are taken from [34]. 

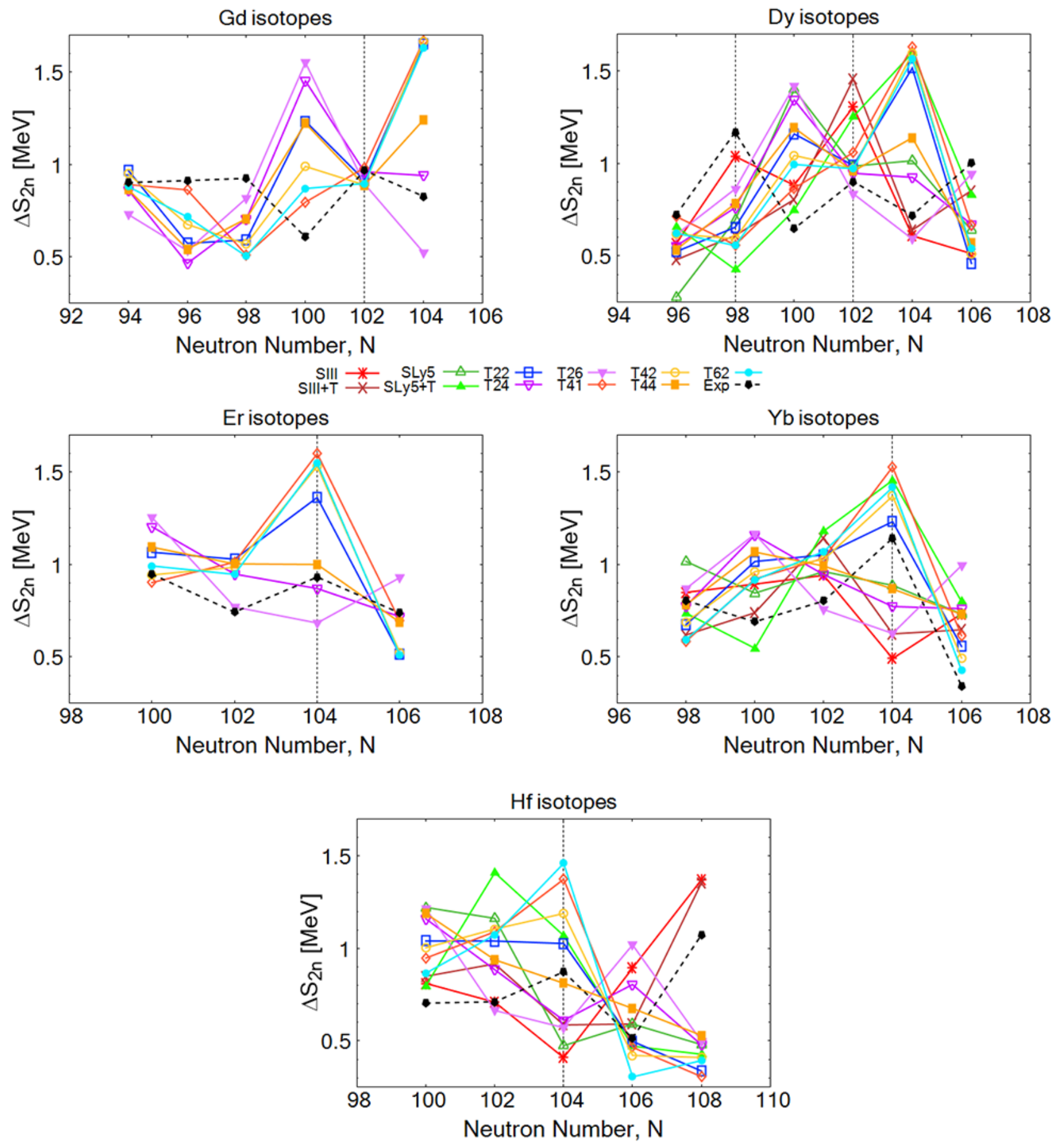

FIG. 2: Two-neutron separation energy differential for ${ }_{64} \mathrm{Gd},{ }_{66} \mathrm{Dy},{ }_{68} \mathrm{Er},{ }_{70} \mathrm{Yb}$ and ${ }_{72} \mathrm{Hf}$ isotopes as a function of neutron number. 

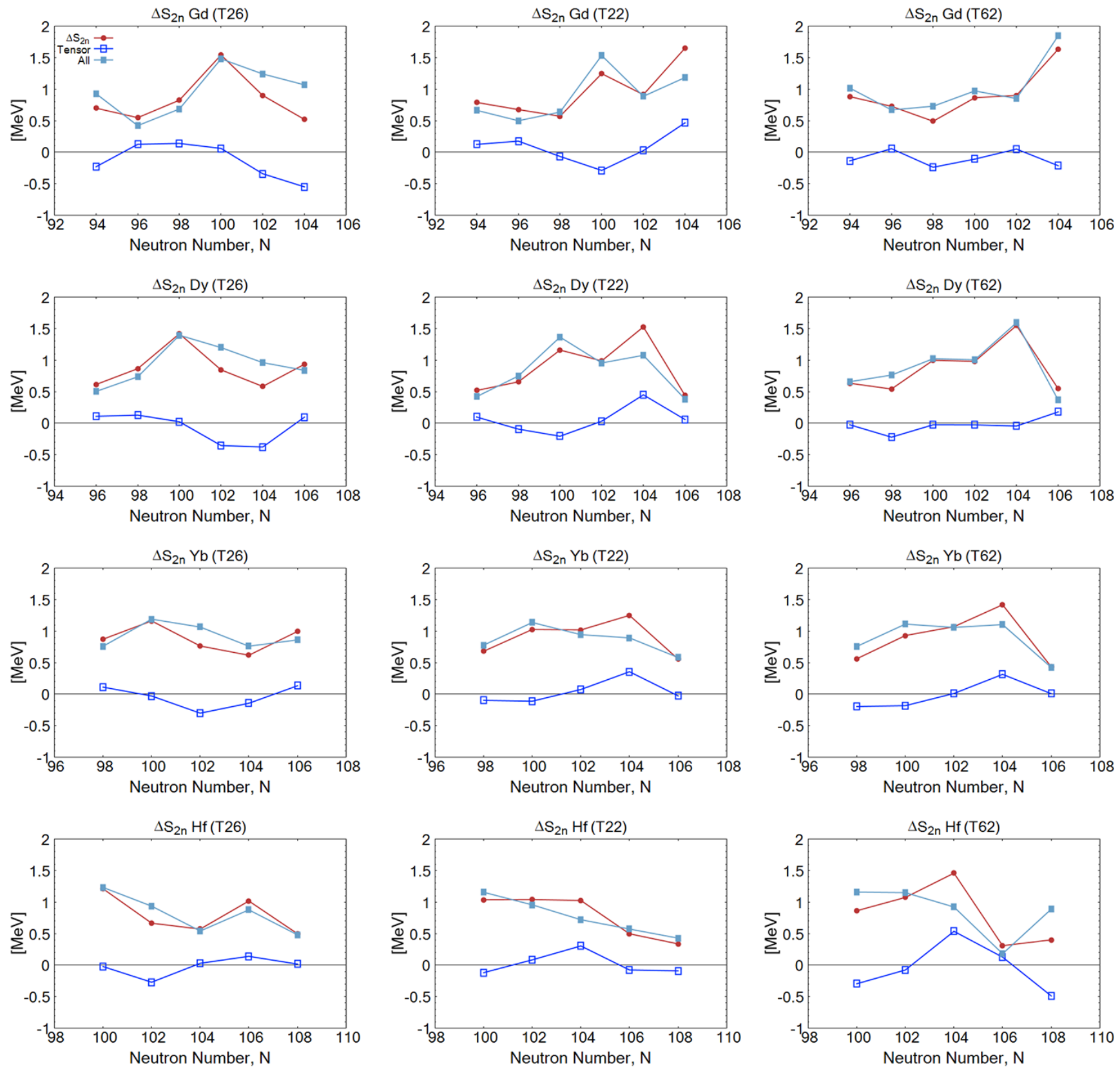

FIG. 3: Contributions of non-tensor and tensor components to the $\Delta S_{2 n}$. 


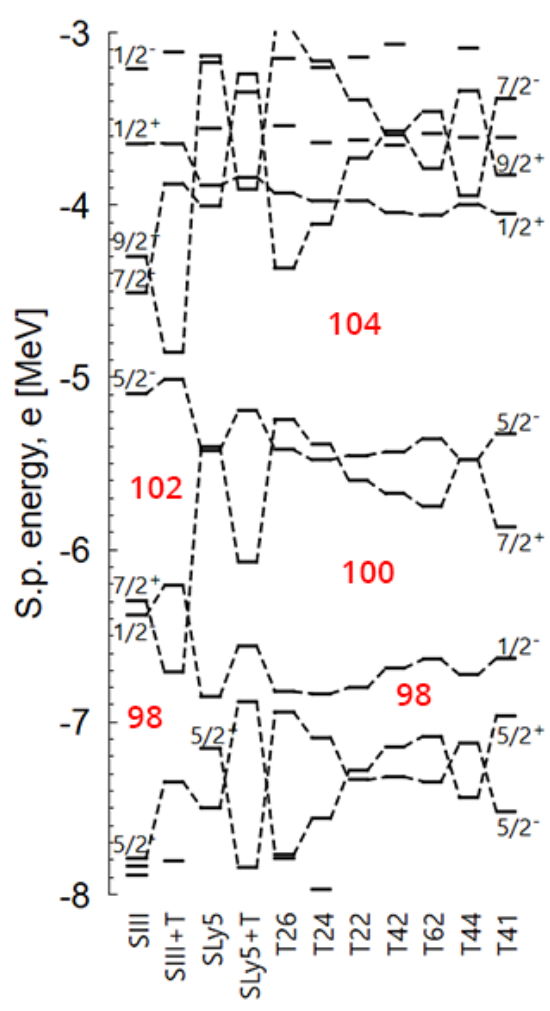

(a) Dy-164

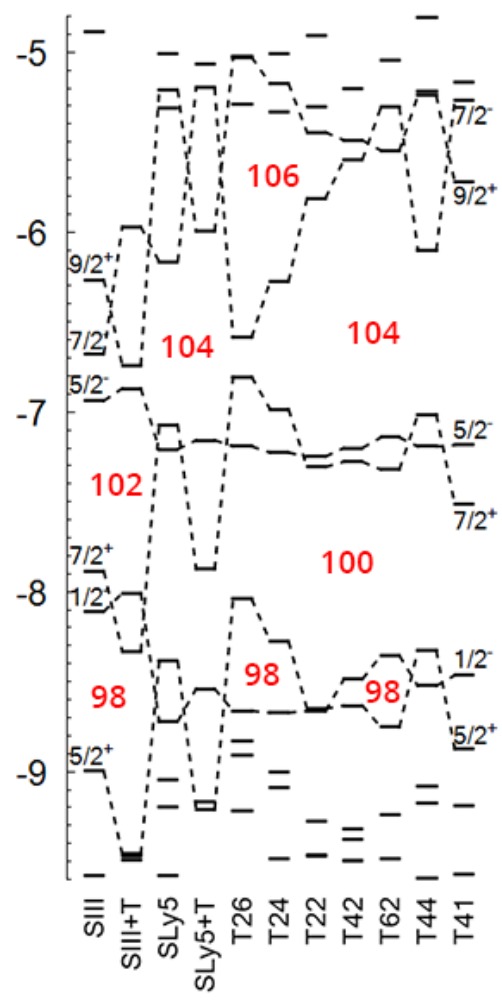

(b) Yb-174

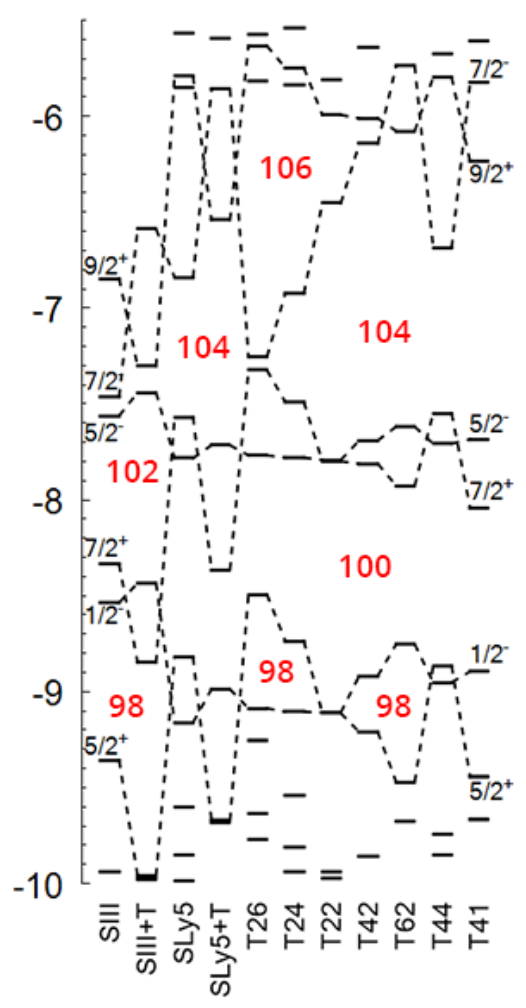

(c) Hf-176

FIG. 4: Neutron single-particle (s.p) spectra for (a)Dy-164, (b)Yb-174 and (c)Hf-176 as a function of Skyrme parameteriazations. 